\title{
Update on Therapeutic Strategy in Lung Carcinoids*
}

\author{
Sara Pusceddu ${ }^{1 \#}$, Milena Vitali ${ }^{1}$, Eva Haspinger ${ }^{1}$, Luca Tavecchio ${ }^{2}$, Riccardo Giovannetti ${ }^{2}$, \\ Andrea Bille ${ }^{2}$, Laura Concas ${ }^{1}$, Marina Garassino ${ }^{1}$, Massimo Milione ${ }^{3}$, \\ Filippo de Braud ${ }^{1}$, Roberto Buzzoni ${ }^{1}$
}

\begin{abstract}
${ }^{1}$ Medical Oncology Unit, Fondazione IRCCS-Istituto Nazionale dei Tumori, Milan, Italy; ${ }^{2}$ Thoracic Surgery Unit, Fondazione IRCCS-Istituto Nazionale dei Tumori, Milan, Italy; ${ }^{3}$ Pathology Unit, Fondazione IRCCS—Istituto Nazionale dei Tumori, Milan, Italy.

Email: "sara.pusceddu@istitutotumori.mi.it
\end{abstract}

Received November $4^{\text {th }}$, 2013; revised November $27^{\text {th }}$, 2013; accepted December $3^{\text {rd }}, 2013$

Copyright (C) 2013 Sara Pusceddu et al. This is an open access article distributed under the Creative Commons Attribution License, which permits unrestricted use, distribution, and reproduction in any medium, provided the original work is properly cited. In accordance of the Creative Commons Attribution License all Copyrights (C 2013 are reserved for SCIRP and the owner of the intellectual property Sara Pusceddu et al. All Copyright @ 2013 are guarded by law and by SCIRP as a guardian.

\begin{abstract}
An estimated $25 \%$ to $30 \%$ of all neuroendocrine tumors (NETs) have their origin in the bronchial tree and into the lungs. Although lung NETs account for less than $1 \%$ of all pulmonary malignancies, the incidence of these neoplasms has risen precipitously since the mid 1970s. Currently, according to the 2004 World Health Organization categorization, these tumors are separated into 4 subtypes characterized by increasing biologic aggressiveness: low-grade typical carcinoid (TC), intermediate-grade atypical carcinoid (AC), high-grade large-cell neuroendocrine carcinoma (LCNEC) and small-cell carcinoma (SCLC). Surgery is the treatment of choice for typical and atypical carcinoid lung NETs with loco-regional disease. At diagnosis up to $64 \%$ of patients with atypical carcinoid lung NETs present with lymph node metastases, and 5-year survival ranges from $61 \%$ to $88 \%$. In contrast, lymph node metastases are present in fewer than $15 \%$ of typical carcinoid lung NETs, and 5-year survival exceeds $90 \%$. To date, there is no recognized standard of treatment for advanced carcinoid lung NETs. In recent years only two trials reported intriguing results regarding lung NETs: a phase 2 retrospective study of dacarbazine derivative temozolomide and the phase 3, RADIANT-2 trial in advanced NETs. Successful management requires a multidisciplinary team management. This review is restricted to typical/atypical NETs.
\end{abstract}

Keywords: Bronchial; Pulmonary; Carcinoids; Neuroendocrine Tumours

\section{Introduction}

Around 25\% - 30\% of all neuroendocrine tumors (NETs) have their origin in the bronchial tree and into the lungs. Bronchopulmonary (BP) typical carcinoids (TCs) and atypical carcinoids (ACs) are uncommon, representing $1 \%-5 \%$ of all pulmonary malignancies with an incidence rate of 5 - 10 cases per million population per year [1-3].

The incidence of these neoplasms has risen over the past 30 years, and these variants are associated with relatively slow growth, and generally show a favorable outcome [3].

Compared with other non-small cell lung cancers

\footnotetext{
*The authors declare that there is no conflict of interests regarding the publication of this article.

"Corresponding author.
}

(NSCLCs), and high grade lung NETs (SCLC and LCNET), advanced typical or atypical carcinoid lung tumors, are less aggressive, and are characterized by an indolent course. The survival is generally good, with an estimate 5 -year overall survival rate of $61 \%$ to $88 \%$ for ACs and of $90 \%$ for TCs; this explains the relative high prevalence of these tumours [4].

Atypical carcinoids are more likely to have peripheral location, to show lymph node metastases, and are more likely to be tobacco-related [5].

The vast majority of symptomatic patients have symptoms directly involving the bronchopulmonary tree. Typical symptoms of carcinoid lung NETs include obstructive pneumonia, atelectasis, and wheezing which are the results of central airway obstruction due to tumor mass, (see Figure 1).

Peripheral pulmonary carcinoid tumors most often are 
asymptomatic and usually are discovered incidentally [5].

Although NETs of the lung arise from cells capable to produce serotonin and adrenocorticotropin hormones, the hypersecretion of bioactive amines is comparatively rare in typical and atypical lung NETs. The most common hormonal syndrome associated with the bronchial carcinoids is the ectopic adrenocorticotropic hormone (ACTH) syndrome. About $10 \%$ - 15\% of all ACTH-dependent Cushing syndrome is ectopic. There is an equal male to female incidence. It may occur at any age from 5 to 90 years [6]. Bronchial NETs may be part of multiple endocrine neoplasia type 1 syndrome (MEN-1), in 5\% - 15\% of cases [7].

\section{Treatment}

\subsection{Histopathological Features}

NETs of the lung origin comprise of a heterogeneous population of tumors ranging from well-differentiated bronchial NETs to highly malignant and poorly differenttiated small-cell lung cancer (SCLC) and large-cell neuroendocrine carcinoma (LCNEC).

TCs and SCLCs are more frequently found to be centrally located in the lung, while ACs and LCNECs more often show a peripheral localization [4].

Based on the present World Health Organization (WHO) 2004 classification of lung NETs that combined architectural growth patterns of tumor cells (organoid growth versus small-cell diffuse growth), mitotic index and the presence of necrosis (see Table 1); ACs are distinguished from TCs by the demonstration of necrosis and/or presence of 2 - 10 mitoses per $2 \mathrm{~mm}^{2}(10 \times$ high-power field) [4]. In TC, there are $<2$ mitoses per 2 $\mathrm{mm}^{2}$ (10 $\times$ high-power field), no necrosis is present and the tumor is $>0.5 \mathrm{~cm}$ in diameter. Histological subtype and nodal status are the most important prognostic factors (see Figure 2-3) [4].

Up to $35 \%$ - $64 \%$ of patients with atypical carcinoid lung NETs present with lymph node metastases, and 5 -year survival ranges from $61 \%$ to $88 \%$. In contrast, lymph node metastases are present in fewer than $15 \%$ of cases of typical carcinoid lung NETs, and 5-year survival exceeds $90 \%$ [4].

\subsection{Biochemical Evaluation and Radiological Assessment}

Biochemical evaluation of well differentiated lung NETs include plasma chromogranin A, plasma-neuron-specific

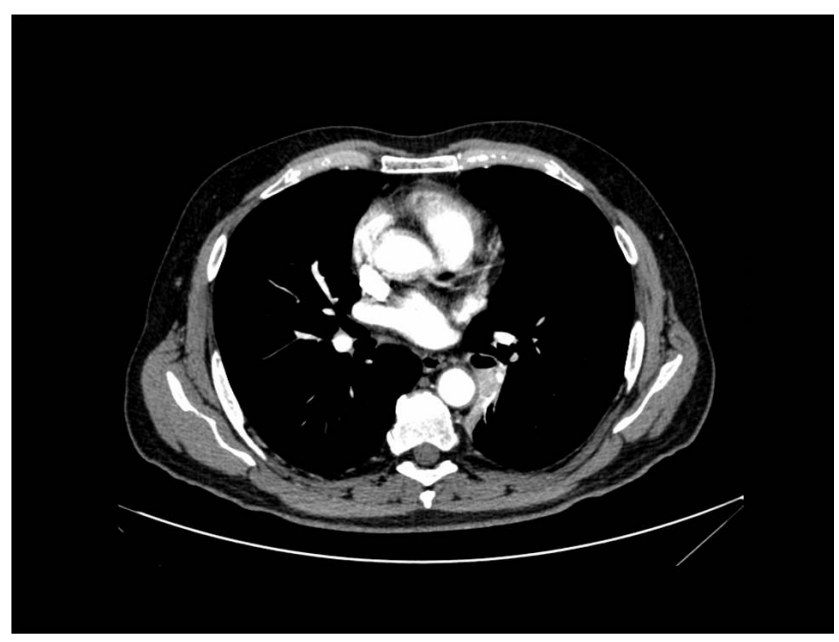

Figure 1. Computerized tomographic study of atypical carcinoid lung tumor of the left mainstem bronchus and resultant lung atelectasis.

Table 1. Neuroendocrine tumors of the lung from travis WD classification.

\begin{tabular}{|c|c|c|c|}
\hline Histological type & Morphology & Necrosis & Mitotic count \\
\hline $\mathrm{TC}$ & Carcinoid morphology $<0.5 \mathrm{~cm}$ & absent & $<2 / 10 \mathrm{HPF}$ \\
\hline $\mathrm{AC}$ & Carcinoid morphology & present focal & $2-9 / 10 \mathrm{HPF}$ \\
\hline LCNEC & $\begin{array}{l}\text { Neuroendocrine morphology (organoid nesting, } \\
\text { palisanding, rosettes, trabeculae }\end{array}$ & present (extensive) & $>9 / 10 \mathrm{HPF}$ \\
\hline SCLC & $\begin{array}{l}\text { Scant cytoplasm, finely granular nuclear chromatin, } \\
\text { absent or faint nucleoli }\end{array}$ & present (extensive) & $>50 / 10 \mathrm{HPF}$ \\
\hline
\end{tabular}

TC: typical carcinoid, AC: atypical carcinoid, LCNEC: large cell neuroendocrine carcinoma, SCLC: small cell lung cancer, HPF: high power fields. 


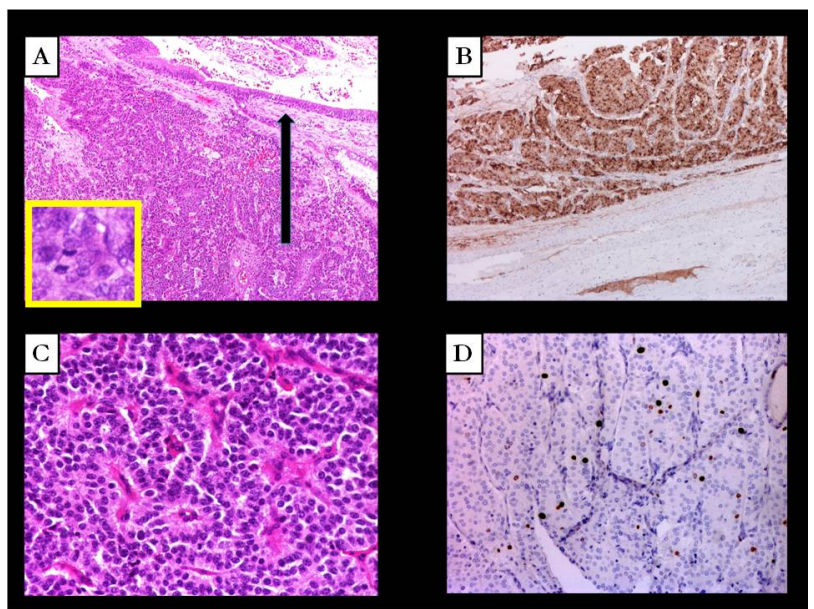

Figure 2. Pulmonary atypical carcinoid primary tumor: (A) well-differentiated neuroendocrine tumor lung in contact with a bronchial structure (black arrow). Mitotic index: three mitosis in $10 \mathrm{HPF}$ (example of mitosis in Figure A yellow box). The tumor is characterized by "pseudosettiform" structures (C) and showing diffuse cytoplasmic positivity for synaptophysin and dot like for chromogranin-A (B). The proliferation index assessed by MIB-1/Ki-67 is $3.5 \%$ (D).

enolase (NSE), and in selected cases with carcinoid syndrome, dU-5-hydroxy indol-acetic acid. In patients with Cushing's disease and acromegaly, plasma ACTH and urine cortisol, and plasma GHRH and insulin growth factor (IGF-I) can be evaluated respectively [4].

Computed tomography (CT) scanning of the chest may suggest a diagnosis of lung NETs. Around $10 \%$ of patients will have multiple lesions and calcifications are evident in up to $30 \%$ of the tumours [7].

Bronchoscopy, if necessary with additional endoscopic ultrasonography with biopsies, is the best procedure to detect and to diagnose central bronchial NETs [7].

Since $80 \%$ of typical bronchial well differentiated carcinoids (TCs and ACs) express somatostatin receptors [8]. The octreotide scan or octreoscan ${ }^{\circledR}$, called also somatostatin receptor scintigraphy (SRS), is used to detect carcinoids; good results were reported with octreotide-derivatives labelled with 111In-pentetreotide for single photon emission computed tomography (SPECT) due to its high affinity to somatostatin type receptor 2 and 5 (SSTR-2 and SSTR-5), mainly expressed in both TCs and ACs [9]. The overall sensitivity and specificity in detection is as high as $93 \%$ and $87 \%$, respectively [10].

New radiopharmaceuticals, labeled with Gallium-68 with an improved biodistribution and increased tumour uptake, have been synthesized for PET/CT combining imaging techniques which leads to 68Ga-DOTATOC PET and 68Ga-DOTATATE PET/CT [11,12].

These last techniques seem to be superior to standard fluoro-deoxy-glucose positron emission tomography (FDG-PET/TC), in the detection of well-differentiated

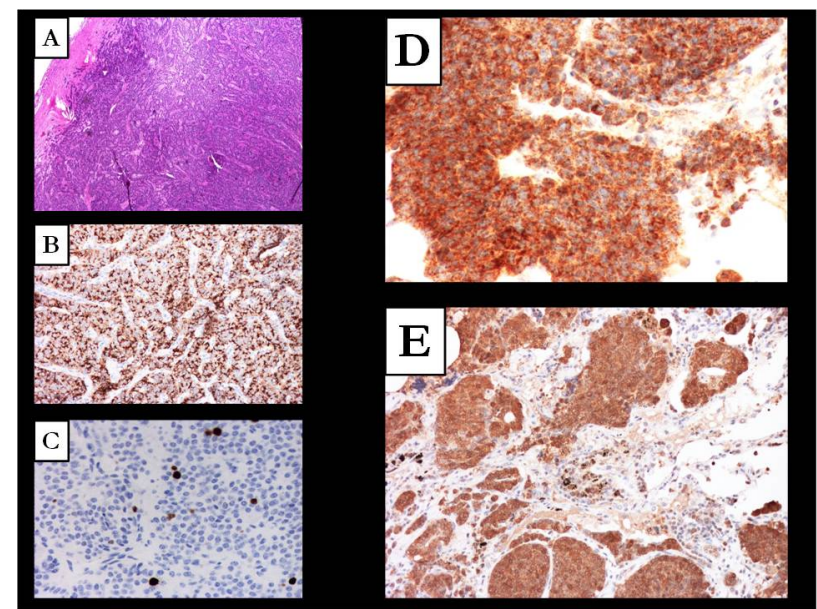

Figure 3. Lymph node metastasis from pulmonary atypical carcinoid: the lymph node is completely replaced by the tumor (A). The lesion presents nodal diffuse cytoplasmic positivity for Synaptophysin and dot-like for Chromogranin A (B). The proliferation index assessed by MIB-1/Ki-67 is $3.7 \%$ (C). Positivity for $\mathrm{m}$-Tor (D) and 4EBP1 (E) is intense and widespread with positive internal controls.

neuroendocrine tumours because of the higher sensitivity in low-grade tumour.

68 Ga-DOTATATE has superior affinity and uptake at the SSTR-2, and combined PET and CT imaging with this tracer has superior spatial resolution compared with conventional 111In-pentetreotide imaging, and actually it is the most sensitive and most accurate imaging techniques to detect small receptor-positive BP carcinoid lesions [13].

Staging with FDG-PET is recommended only for more aggressive bronchial NETs such as LCNEC and SCLC. The role of FDG-PET imaging is not well defined for BP carcinoids, for the high rate of FDG-PET false negative due to low grade, low mitotic count and often small dimension [12,14].

\subsection{Management of Locally Advanced Disease}

Surgical resection remains the treatment of choice for patients with pulmonary carcinoids [7].

Bronchoscopic laser excision of intraluminal typical bronchial NETs should be considered as a suboptimal treatment and reserved for inoperable patients or performed as a preoperative disobliterating procedure [15].

The surgical techniques of choice are lobectomy or sleeve resection. Pneumonectomy should be avoided except in selected cases. Limited resection should be performed for known carcinoid tumors. Unknown lung lesions should undergo wedge excisional biopsy. If intraoperative frozen section is consistent with carcinoid and the margins are negative, mediastinal lymph node dissection should be performed. If the patient is node negative then completion lobectomy is not required. In 
node-positive patients with adequate pulmonary reserve, lobectomy should be performed regardless of histology. If atypical features are found during permanent pathologic evaluation then interval completion lobectomy may be considered in good-risk patients [15].

Systemic nodal dissection should be performed since lymphonodal metastases may be present in up to $15 \%$ of cases in TC and $>50 \%$ in AC. TCs exhibit a good prognosis with a 5-year survival rate in $92 \%$ - $100 \%$ of cases. ACs are regarded as intermediate in grade and are associated with a poor prognosis and a 5-year survival rates of $61 \%-88 \%$ [ 4$]$.

\subsection{Management of Advanced/Metastatic Disease}

There is no recognized first-line therapy for advanced carcinoid lung tumours [7].

Cytotoxic treatment combined with surgical resection when indicated, has been the standard for metastatic lung NETs, although the available chemotherapy regimens demonstrate a rather poor effect. A combination of cisplatinum and etoposide is mainly used in high proliferating tumors.

Available chemotherapy regimens for TCs and ACs include a combination of streptozotocin plus 5-fluorouracil/doxorubicin, or capecitabine/oxaliplatin. Temozolomide alone or in combination with bevacizumab, has demonstrated clinical benefit [7].

Ekeblad and colleagues performed a retrospective analysis of 36 patients with histologically confirmed metastatic or inoperable malignant NETs treated with oral temozolomide (100 - $200 \mathrm{mg} / \mathrm{m}^{2} / \mathrm{d}$ for 5 days every 28 days). The study group included 10 pts with TCs and 3 with ATs. After a median follow-up of 7 months (range, 2 - 17 months), $31 \%$ of patients with lung carcinoids had stable disease (SD) and 31\% showed a partial radiologic response (PR) [16].

No chemotherapies randomized trials have been performed that could guide the treatment, and at present, there is no established standard regimen and the role of chemotherapy in BP carcinoids continues to be debated.

For low proliferating tumors, treatment with somatostatin analogs might be an option for functional tumors with clinical symptoms. Treatment with these agents has resulted in $\mathrm{PR}$ in 5\% - 10\%, SD in 30\% - 50\% and symptomatic improvement in $40 \%-60 \%$ of cases. In nonfunctioning tumors, the use of somatostatin analogs is still controversial, but after the PROMID and CLARINET trials indicating antitumor efficacy of octreotide long acting release (LAR) and Lanreotide Autogel drugs in gastroenteropancreatic NETs, it is now also widely accepted for nonfunctioning tumors of other origins. Peptide receptor radiotherapy is an option in patients with tumors that present with a high content of soma- tostatin receptors $[17,18]$.

The molecular biology of the lung carcinoids, generally show an overexpression of EGF (epidermal growth factor) of IGF (insulin-like growth factor) and their receptors. The RAS-RAF-MAP kinase pathway is overacttive, and there is usually overactivity of PI3 kinase and the mTOR pathway. Recent in vitro studies indicate that somatostatin receptors are overexpressed in metastatic typical carcinoid tumors of the lung [8] and that the mTOR is found in most lung NETs with higher expression in typical and atypical carcinoids (see Figure 3). [19].

In addition, a recent preclinical study of the impact of the mTOR inhibitor, everolimus, found that it suppressed the viability of typical and atypical carcinoid lung cells in culture [20]. Thus, there is a theoretical rationale for the use of both Tyrosine-kinase inhibitor (TKIs) and mTOR inhibitors.

Despite Lung NETs are typically underrepresented in clinical trials of NET treatments, recently, a phase 3 RAD001 in Advanced Neuroendocrine Tumors Trial (RADIANT-2) has reported results specific for BP carcinoids NETs [21].

In RADIANT-2, which evaluated the impact of combination therapy with the oral mammalian target of rapamycin (mTOR) inhibitor everolimus and the somatostatin analogue octreotide LAR in patients with advanced NET and carcinoid symptoms; only $6.9 \%$ of patients in the experimental group and $2.3 \%$ of pts in the control group were diagnosed with lung NETs. Patients were randomly assigned to receive octreotide LAR 30 mg intramuscularly every 28 days combined with everolimus $10 \mathrm{mg}$ per day $(\mathrm{N}=216)$ or octreotide LAR plus a placebo $(\mathrm{N}=213)$. Treatment with everolimus combined with octreotide was associated with longer progression-free survival overall: 16.4 months in patients treated with everolimus and octreotide vs 11.3 months in control patients $(P=0.026)$; patients with lung NETs show trend to improve progression free survival with Everolimus plus Octreotide $(P=0.228)$ [21,22].

Based on that, the ongoing RADIANT-4 trial (NCT 01524783) is looking at progressive nonfunctioning NETs, including lung carcinoids where patients are treated with everolimus or placebo. Finally a trial specifically looking at lung NETs, the LUNA trial (NCT 01563354), where patients are randomly assigned to everolimus, the somatostatin analog pasireotide, or the combination of both, is ongoing and is still enrolling patients.

An other trial with biological target therapies, which some preliminary data had been reported from, is the PAZONET trial. The PAZONET trial looked at progressive new endocrine tumors and showed a clinical benefit in $85 \%$ of patients treated with pazopanib, including pa- 
tients with lung carcinoids [23].

However the role of targeted therapies for typical and atypical carcinoid lung NETs remains incompletely defined, with data from relatively few clinical trials to help guide clinical decision making.

\section{Summary and Conclusions}

Diagnosis remains challenging. Proper distinction should be made between well or moderate differentiate subtypes (AT and CT carcinoids) and poorly differentiated forms (LCNC and SCLC).

The role of histology and systematic nodal dissection performed during surgery is the key.

Imaging techniques, like radiolabeled peptide scintigraphy with 111In-pentetreotide or 68Ga-DOTATOC/ DOTATATE PET/CT are useful in well differentiated bronchial NETs rather than LCNEC and SCLC histotypes, where FDG-PET/TC scans are more informative than somatostatin receptor scintigraphy. Surgery is the treatment of choice for typical and atypical carcinoid lung NETs with loco-regional disease. For advanced disease, no standard treatment or therapeutic algoritm is available. Somatostatin analogues may be recommended as first line treatment, while the role of targeted therapy for typical and atypical lung carcinoid remains unclear, with data from relatively few clinical trials to help guide clinical decision making.

Further research is needed to clarify the role of somatostatin analogues, mTOR inhibitors, and other targeted therapies on these rare and clinically challenging tumors.

\section{REFERENCES}

[1] I. M. Modlin, K. D. Lye and M. A. Kidd, "5-Decade Analysis of 13,715 Carcinoid Tumors," Cancer, Vol. 97, No. 4, 2003, pp. 934-959.

[2] W. D. Travis, "Lung Tumours with Neuroendocrine Differentiation,” European Journal of Cancer, Vol. 45, Suppl. 1, 2009, pp. 251-266. http://dx.doi.org/10.1016/S0959-8049(09)70040-1

[3] J. C. Yao, M. Hassan, A. Phan, et al., "One Hundred Years after 'Carcinoid': Epidemiology of and Prognostic Factors for Neuroendocrine Tumors in 35,825 Cases in the United States.” Journal of Clinical Oncology, Vol. 26, No. 18, 2008, pp. 3063-3072.

[4] W. D. Travis "Advances in Neuroendocrine Lung Tumors,” Annals of Oncology, Vol. 21, Suppl. 7, 2010, pp. 65-71. http://dx.doi.org/10.1093/annonc/mdq380

[5] O. Hauso, B. I. Gustafsson, M. Kidd, et al., "Neuroendocrine Tumor Epidemiology: Contrasting Norway and North America," Cancer, Vol. 113, No. 10, 2008, pp. 2655-2664.

[6] A. M. Isidori, G. A. Kaltsas and A. B. Grossman, "Ectopic ACTH Syndrome,” Frontiers of Hormone Research, Vol. 35, 2006, pp. 143-56. http://dx.doi.org/10.1159/000094323

[7] K. Öberg, P. Hellman, P. Ferolla, et al., "Neuroendocrine Bronchial and Thymic Tumors: ESMO Clinical Practice Guidelines for diagnosis, Treatment and Follow-Up. ESMO Guidelines Working Group,” Annals of Oncology, Vol. 23, Suppl. 7, 2012, pp. 120-123.

[8] L. Righi, M. Volante, V. Tavaglione, et al., "Somatostatin Receptor Tissue Distribution in Lung Neuroendocrine Tumours: A Clinicopathologic and Immunohistochemical Study of 218 'Clinically Aggressive' Cases,” Annals of Oncology, Vol. 21, No. 3, 2010, pp. 548-555. http://dx.doi.org/10.1093/annonc/mdp334

[9] M. Papotti, S. Croce, M. Bello, et al., "Expression of Somatostatin Receptor Types 2, 3 and 5 in Biopsies and Surgical Specimens of Human Lung Tumours. Correlation with Preoperative Octreotide Scintigraphy," Virchows Archiv, Vol. 439, 2001, pp. 787-797.

[10] I. M. Modlin, K. Oberg, D. C. Chung, et al., "Gastroenteropancreatic Neuroendocrine Tumours," The Lancet Oncology, Vol. 9, No. 1, 2008, pp. 61-72. http://dx.doi.org/10.1016/S1470-2045(07)70410-2

[11] I. J. Virgolini, M. Gabriel, E. von Guggenberg, et al., "Role of Radiopharmaceuticals in the Diagnosis and Treatment of Neuroendocrine Tumours," European Journal of Cancer, Vol. 45, Suppl. 1, 2009, pp. 274-291. http://dx.doi.org/10.1016/S0959-8049(09)70042-5

[12] P. Antunes, M. Ginji, H. Zhang, et al., "Are Radiogallium-Labelled DOTA-Conjugated Somatostatin Analogues Superior to Those Labelled with Other Radiometals," European Journal of Nuclear Medicine and Molecular Imaging, Vol. 34, No. 7, 2007, pp. 982-993. http://dx.doi.org/10.1007/s00259-006-0317-x

[13] A. R. Haug, R. Cindea-Drimus, C. J. Auernhammer, et al., "Neuroendocrine Tumor Recurrence: Diagnosis with 68Ga-DOTATATE PET/CT,” Radiology, Vol. 20, 2013.

[14] T. Binderup, U. Knigge, A. Loft, et al., "Functional Imaging of Neuroendocrine Tumors: A Head-to-Head Comparison of Somatostatin Receptor Scintigraphy, 123IMIBG Scintigraphy, and 18F-FDG PET,” Journal of Nuclear Medicine, Vol. 51, No. 5, 2010, pp. 704-712. http://dx.doi.org/10.2967/jnumed.109.069765

[15] W. W. de Herder, V. Mazzaferro, L. Tavecchio, et al., "Multidisciplinary Approach for the Treatment of Neuroendocrine Tumors,” Tumori, Vol. 96, No. 5, 2010, pp. 833-846.

[16] S. Ekeblad, A. Sundin, E. T. Janson, et al., "Temozolomide as Monotherapy is Effective in Treatment of Advanced Malignant Neuroendocrine Tumors,” Clinical Cancer Research, Vol. 13, No. 10, 2007, pp. 2986-2991. http://dx.doi.org/10.1158/1078-0432.CCR-06-2053

[17] A. Rinke, H. H. Müller, C. Schade-Brittinger, et al., "Placebo-Controlled, Double-Blind, Prospective, Randomized Study on the Effect of Octreotide LAR in the Control of Tumor Growth in Patients with Metastatic Neuroendocrine Midgut Tumors: A Report from the PROMID Study Group. PROMID Study Group,” Journal of Clinical Oncology, Vol. 27, No. 28, 2009, pp. 46564663.

[18] M. Caplin, P. Ruszniewski, M. Pavel, et al., “A Random- 
ized Double-Blind Placebo-Controlled Study of Lanreotide Antiproliferative Response in Patients with Enteropancreatic Neuro Endocrine Tumours (CLARINET)," 17th ECCO-38th ESMO-32nd ESTRO European Cancer Congress, Amsterdam, Vol. 49, No. 3, 2013, Abstract E17-7103.

[19] L. Righi, M. Volante, I. Rapa, et al., "Mammalian Target of Rapamycin Signaling Activation Patterns in Neuroendocrine Tumors of the Lung," Endocrine-Related Cancer, Vol. 17, No. 4, 2010, pp. 977-987. http://dx.doi.org/10.1677/ERC-10-0157

[20] M. C. Zatelli, M. Minoia, C. Martini, et al., "Everolimus as a New Potential Antiproliferative Agent in Aggressive Human Bronchial Carcinoids,” Endocrine-Related Cancer, Vol. 17, No. 3, 2010, pp. 719-729. http://dx.doi.org/10.1677/ERC-10-0097

[21] M. E. Pavel, J. D. Hainsworth, E. Baudin, et al., "Ever- olimus Plus Octreotide Long-Acting Repeatable for the Treatment of Advanced Neuroendocrine Tumours Associated with Carcinoid Syndrome (RADIANT-2): A Randomised, Placebo-Controlled, Phase 3 Study,” Lancet, Vol. 378, No. 9808, 2011, pp. 2005-2012.

[22] N. Fazio, D. Granberg, A. Grossman, et al., "Everolimus Plus Octreotide Long-Acting Repeatable in Patients with Advanced Lung Neuroendocrine Tumors: Analysis of the Phase 3, Randomized, Placebo-Controlled RADIANT-2 Study,” Chest, Vol. 143, No. 4, 2013, pp. 955-962. http://dx.doi.org/10.1378/chest.12-1108

[23] E. Gande, et al., "sVEGFR2 and Circulating Tumor Cells to Predict for the Efficacy of Pazopanib in Neuroendocrine Tumors (NETs): PAZONET Subgroup Analysis," Journal of Clinical Oncology, Vol. 31, Suppl. 15, 2013. 